\title{
UK internet antenatal dietary advice: a content accuracy and readability analysis
}

\author{
Anne Sidnell ${ }^{1}$ and Penelope Nestel ${ }^{2}$ \\ ${ }^{1}$ The Nutrition Society, London, United Kingdom and \\ ${ }^{2}$ University of Southampton, Southampton, United Kingdom
}

\section{Abstract}

Nutrition in pregnancy is important for the health of the mother and infant. Suboptimal maternal nutrition can result in poor pregnancy outcomes for the infant such as low birth weight, hypovitaminosis D, and neural tube defects. Theinternet is a popular source of dietary advice in pregnancy, but there are no regulations to control the quality of information provided,and poor accuracy and readability can hinder its usefulness.

The purpose of this study was to examine the accuracy and readability of online pregnancy-related diet and nutrition information regardless of geographical origin when searching from the UK. The accuracy was assessed against UK government advice and the readability using theFlesch Readability Ease (FRE) tool. Three commonly searched themes were examined;foods to avoid, foods to eat, and supplements.

130 web pages were rated for accuracy and readability. Descriptive and non-parametric tests were used and Spearman's Correlation used to explore the relationship between accuracy and readability.

Eighty-three $(64 \%)$ web pages contained accurate and inaccurate advice, $23(18 \%)$ were accurate, $21(16 \%)$ were inaccurate, and three $(2 \%)$ lacked relevant advice. The median percentage accuracy of all advice was 83 (LQ, UQ) (48,100). Eighty-seven pages $(67 \%)$ scored below the recommended FRE for public web pages. The median FRE was 55 (46, 61) defined as 'fairly difficult'. There was a weak positive correlation between accuracy and readability of web pages, rho $=0.241, p=0.006$. There was no significant difference in accuracy of web page by theme. Readability of web pages on supplements was more difficult than foods to avoid and foods to eat. Web pages from the UK $(58 \%)$ were more accurate, difference of median $33(16,40) \mathrm{p}<0.001$ and more readable, difference of median $9.1(5.0,13.1) \mathrm{p}<0.001$ than those from outside the UK. Web pages from commercial sources $(76 \%)$ were significantly less accurate than those from non-for-profit organisations, difference of median $-8(-29,0.00) \mathrm{p}=0.019 .27 \%$ of web pages originated from the US. Dietary advice for pregnancy from the US differs from that in the UK.

Despite the popularity of internet searching, much pregnancy-related dietary advice online is inaccurate and difficult to comprehend. Contradictory advice may interrupt the process of making improvements to diet in pregnancy, and result in negative health outcomes for mother and infant. Healthcare professionals can support women by guiding them to accurate advice, which should only be provided by those qualified in nutrition or dietetics.

\section{Conflict of Interest}

There is no conflict of interest 